\title{
Risk of Confusion in the Prescription and Administration of Generic Drugs
}

\author{
Gerald Wendelin ${ }^{1 *}$, Sabine Löffler ${ }^{1}$, Elisabeth Thierrichter ${ }^{1}$, Astrid Sonnleitner ${ }^{1}$, Wolfgang Schwinger ${ }^{2}$ \\ From Safety in hospitals: from strategy to implementation Annual Scientific Meeting 2015 \\ Graz, Austria. 29-30 September 2015
}

\section{Background}

In Austria physicians are legally obligated to use trade names instead of active components when prescribing drugs. The increasing number of generics and short term changes in the availability of generics due to reduced inventories of manufacturers and dealers force physicians to frequent changes in their prescriptions. Frequent changes are associated with an increased risk of confusion in prescription and administration of generics. For secure prescription and administration of generics it is necessary that physicians have access to valid information about equivalence and daily disposability of generics.

\section{Material and methods}

An IT-based process for reducing the risk of confusion in prescribing and dispensing of generic drugs, which was developed at the University Department of Pediatrics and Adolescent Medicine Graz, will be presented.

\section{Results}

The ward nurse carries a continuously updated list of available drugs. This list contains two columns. In the left column, originator drugs available on the ward are listed. In the right column equivalent generic drugs are listed. Equivalent generic drugs were defined as drugs with equivalent ingredients and identical in terms of expected effects and side effects in comparison to the original product.

The list is validated and approved by physicians.

The list, shared by various clinical divisions, is fed into an Internet database. Access to the database is via an icon on the desktop of all computers of the clinic.
The physicians can only prescribe drugs that are available according to the current list. With proper list management the available drugs are the generic drugs cited in the right column, or if this column is empty, the originator drug in the left column.

The nursing staff is not entitled to dispense drugs if the name does not match the doctor's prescription. In such cases the responsible physician must be contacted and made aware of the unenforceability of the prescription.

\section{Conclusions}

For the safe prescription and administration of generic drugs a clearly defined process is required, which ensures that only currently available drugs are prescribed. This should reduce confusion and limit the number of times nursing staff need to contact doctors because of drug prescriptions.

\section{Authors' details \\ ${ }^{1}$ Department of Pediatrics and Adolescent Medicine, Medical University of Graz, Graz, Austria. ${ }^{2}$ Department of Pediatrics and Adolescent Medicine, Division of Paediatric Hemato-Oncology, Medical University of Graz, Graz, Graz, Austria.}

Published: 30 October 2015

doi:10.1186/2056-5917-1-S1-A27

Cite this article as: Wendelin et al:: Risk of Confusion in the Prescription and Administration of Generic Drugs. Safety in Health 2015 1(Suppl 1): A27.

\footnotetext{
* Correspondence: gerald.wendelin@medunigraz.at

'Department of Pediatrics and Adolescent Medicine, Medical University of

Graz, Graz, Austria

Full list of author information is available at the end of the article
} 